\title{
Comparative effectiveness of in-person vs. remote delivery of the Common Elements Treatment Approach for addressing mental and behavioral health problems among adolescents and young adults in Zambia: Protocol of a three-arm randomized controlled trial
}

Caleb Figge ( $\nabla$ cfigge1@jh.edu ) Johns Hopkins Bloomberg School of Public Health https://orcid.org/0000-0003-4953-6191 Jeremy C. Kane Columbia University Mailman School of Public Health

Stephanie Skavenski Johns Hopkins University Bloomberg School of Public Health Emily Haroz Johns Hopkins University Bloomberg School of Public Health

Mwamba Mwenge CIDRZ: Center for Infectious Disease Research in Zambia

Saphira Mulemba CIDRZ: Center for Infectious Disease Research in Zambia

\section{Luke R. Aldridge} Johns Hopkins University Bloomberg School of Public Health

Michael J. Vinikoor

University of Alabama at Birmingham

\section{Anjali Sharma}

CIDRZ: Center for Infectious Disease Research in Zambia

\section{Sachi Inoue}

Harvard University T H Chan School of Public Health

Ravi Paul

University of Zambia

Francis Simenda

Zambia Ministry of Health

Kristina Metz

Johns Hopkins University Bloomberg School of Public Health Carolyn Bolton 
CIDRZ: Center for Infectious Disease Research in Zambia

\section{Christopher Kemp}

University of Washington School of Public Health

\section{Samuel Bosomprah}

CIDRZ: Center for Infectious Disease Research in Zambia

\section{Izukanji Sikazwe}

CIDRZ: Center for Infectious Disease Research in Zambia

\section{Laura K. Murray}

Johns Hopkins University Bloomberg School of Public Health

\section{Research Article}

Keywords: Global mental health, Adolescents, Randomized controlled trial, Telehealth, Zambia, Implementation science

Posted Date: February 25th, 2022

DOI: https://doi.org/10.21203/rs.3.rs-1321327/v1

License: (c) (1) This work is licensed under a Creative Commons Attribution 4.0 International License. Read Full License 


\section{Abstract \\ Background}

In low- and middle-income countries (LMIC) there is a substantial gap in treatment of mental and behavioral health problems, which is particularly detrimental to adolescents and young adults (AYA). The Common Elements Treatment Approach (CETA) is an evidence-based, flexible, transdiagnostic intervention delivered by lay counselors to address comorbid mental and behavioral health conditions, though its effectiveness has not yet been tested among AYA. This paper describes the protocol for a randomized controlled trial that will test the effectiveness of traditional in-person delivered CETA and a telehealth-adapted version of CETA (T-CETA) in reducing mental and behavioral health problems among AYA in Zambia. Non-inferiority of T-CETA will also be assessed.

\section{Methods}

This study is a Hybrid Type 1 three-arm randomized trial to be conducted in Lusaka, Zambia. Following an apprenticeship model, experienced non-professional counselors in Zambia will be trained as CETA trainers using a remote, technology-delivered training method. The new CETA trainers will subsequently facilitate technology-delivered trainings for a new cohort of counselors recruited from community-based partner organizations throughout Lusaka. AYA with mental and behavioral health problems seeking services at these same organizations will then be identified and randomized to 1) in-person CETA delivery, 2) telehealth-delivered CETA (T-CETA), or 3 ) treatment as usual (TAU). In the superiority design, CETA and T-CETA will be compared to TAU and using a non-inferiority design, T-CETA will be compared to CETA, which is already evidence-based in other populations. At baseline, post-treatment (approximately 3-4 months post-baseline), and 6 months post-treatment (approximately 9 months post-baseline) we will assess as primary outcomes client trauma symptoms, internalizing symptoms, and externalizing behaviors and as secondary outcomes client substance use, aggression, violence, and health utility. CETA trainer and counselor competency and cost-effectiveness will also be measured as secondary outcomes. Mixed methods interviews will be conducted with trainers, counselors, and AYA participants to explore the feasibility, acceptability, and sustainability of technology-delivered training and T-CETA provision in the Zambian context.

\section{Discussion}

Adolescents and young adults in LMIC are a priority population for treatment of mental and behavioral health problems. Technology-delivered approaches to training and intervention delivery can expand the reach of evidence-based interventions. If found effective, CETA and T-CETA would help address a major barrier to the scale-up and sustainability of mental and behavioral treatments among AYA in LMIC. 


\section{Trial Registration:}

This protocol was prospectively registered with www.clinicaltrials.gov (NCT03458039, May 10, 2021). https://clinicaltrials.gov/show/NCT03458039.

\section{Background}

The quality and availability of mental and behavioral health care in low and middle-income countries (LMIC) is significantly deficient [1-3]. The high need and inadequate resourcing for mental health results in a substantial treatment gap $[1,2,4]$ that directly contributes to ongoing violation of human rights, abuse and neglect, reduced adherence to medical regimens, long-term disability, ill-health, lower economic productivity, and increased mortality [5-9].

Adolescents and young adults (AYA) in LMIC are in critical need of access to mental and behavioral healthcare. Mental and behavioral health problems account for up to $30 \%$ of disability-adjusted life years before the age of 30 [10]. AYA are disproportionately affected by the HIV epidemic, accounting for $30 \%$ of all new HIV infections [11]. In Zambia, the location of the current study, AYA are at high risk of HIV infection, poverty, experienced violence (including sexual and gender-based violence), and unemployment $[12,13]$. The combination of past and continuing stressors can cause stress-related problems including maladaptive behaviors, emotional and/or behavioral dysregulation, cognitive challenges, substance use/abuse, aggression, risky sexual behavior, and difficulties with functioning [1,15-21], which in turn, increase risk for a range of poor outcomes, including HIV/AIDS risk, substance abuse, and lower economic productivity $[8,22-24]$.

The body of research and policy supporting the effectiveness of mental health treatments is growing rapidly [25-30]. Research shows that certain mental health treatments are effective, acceptable, feasible and can be implemented in LMIC with positive clinical outcomes using an apprenticeship model and a task-sharing approach where lay providers with limited formal mental health training function as counselors [25-28, 30-32]. Despite this evidence, the wide-scale update and sustainability of nonprofessional delivered interventions in LMIC is virtually non-existent.

The Common Elements Treatment Approach (CETA) is an intervention that is flexible, transdiagnostic, and delivered by lay counselors or non-professionals in LMIC to address comorbid mental and behavioral health problems [31]. CETA is grounded in cognitive behavioral therapy elements common to evidencebased treatments (EBTs) for trauma, behavioral problems, anxiety, and depression [33]. This approach allows a counselor to decide on which element(s), order, and dose are most appropriate for each client based on presentation. Randomized controlled trials (RCT) have demonstrated the effectiveness of CETA for a range of mental health, substance use, and behavioral issues among adults in a variety of lowresource settings [34-39]. In a non-randomized study in Ethiopia, CETA improved mental and behavioral health problems improved among youth [40], however, CETA has not yet been evaluated among adolescents in a fully powered randomized trial. 
Trainings for EBTs, such as CETA, are typically provided by an "expert" who usually is a Ph.D. level expatriate with extensive experience and EBT purveyor-approved. After the trainings, the "expert" continues EBT supervision and coaching to ensure skill transfer and fidelity [41]. Multiple studies completed on EBTs in LMIC have demonstrated the use of expert-delivered training with the apprenticeship model is feasible and effective $[25,26,28,30]$. However, scale-up and sustainability efforts of EBTs are nearly impossible when utilizing an expert-delivered training approach since experts are scarce, costly, have limited time, usually require a translator, and often need to travel long distances for on-site trainings, something that was particularly problematic during the COVID-19 pandemic.

A Train the Trainer (TTT) strategy is a specific form of capacity building designed to provide trainees with the necessary knowledge and skills to become trainers themselves - and, arguably, sustain a workforce over time. The TTT model has been effectively used in a diverse range of disciplines (e.g., education, health care) [42-45] and has involved multiple types of trainees (e.g. direct care counselors, parents, or teachers) depending on the complexity of skills being taught [46-48]. However, there is limited research and few rigorous studies on whether TTT efforts achieve their desired level of change, particularly with mental health EBT [49]. Research also suggests that technology-based training approaches (i.e., video and audio-based instruction) may have potential to result in comparable learning to face-to-face instruction across LMIC settings [50-53]. Thus, applying technology-based training approaches to TTT may increase scalability of EBTs $[54,55]$.

In addition to technology-based training of trainers, technology-based delivery of health interventions has been identified as a potential strategy to expand mental healthcare access in LMIC settings [56, 57]. However, most of the evidence showing efficacy of technology-based mental health delivery has been conducted in high-income countries (HIC) with only discussion of its implications for implementation in LMIC $[57,58]$. The majority of studies in this area have focused on videoconferencing, with documented high satisfaction and acceptability of tele-mental health delivery for children and AYA that are underserved or located in rural areas [59-62]. During the COVID-19 pandemic, some mental health practitioners in upper-income countries transitioned almost universally to teletherapy. In various LMIC settings, tele-mental health delivery has been shown to be feasible [63-68] and similar to HIC contexts, and satisfactory comparable to in-person visits [64]. However, there is limited evidence on treatment effectiveness and symptom reduction with technology-based delivery relative to in-person implementation in limited-resource settings [64].

Tele-mental health delivery methods reduce common barriers to treatment access and adherence, including time, financial, and transportation demands, among hard-to-reach populations, particularly during public health crises. Given the rapid increase to access to mobile technology in recent years, telemental health delivery has become a viable option. In January 2021, there were over 19 million mobile phone subscriptions in Zambia, an equivalent of about $104 \%$ of the population[69]. In addition, previous technology-based healthcare delivery systems have found high user acceptance and increased adoption rates of mobile technology that is low-cost, readily available, culturally sensitive, and easy to operate [66]. Exploring technology options and models for treatment delivery is critical for providing and scaling up 
care in hard-to-reach populations and reducing the treatment gap in LMIC settings [65, 70]. Together, techdelivered training and telehealth delivery can reduce the need for in-person logistic demands across the training and treatment cascade at trainer, counselor, and client levels, increasing the feasibility, sustainability, and cost-effectiveness of evidence based mental health care provision in LMIC settings.

Given the substantial mental and behavioral health treatment gap for AYA in LMIC and the potential for technology to improve the delivery and sustainability of EBTs, research into the effectiveness and feasibility of technology-based approaches to training and intervention delivery is warranted. In this paper, we describe the protocol for a randomized controlled trial to test the comparative effectiveness of traditional in-person CETA and telehealth-delivered CETA (T-CETA) featuring lay counselors trained by trainers through a technology-based TTT approach in reducing mental and behavioral health problems among AYA in an urban setting of Zambia.

\section{Methods}

\section{Study Setting}

All study procedures will take place in Lusaka, Zambia. Participants will be recruited from communitybased organizations that provide health, education, and social services to AYA in Lusaka. Organizations with access to AYA populations and with existing counseling/mental health services were selected, as they will serve as prime CETA and T-CETA service delivery points, should the study demonstrate feasibility and effectiveness in this population. Selected organizations include a combination of local and international non-governmental organizations (NGOs) and government health clinics. Heterogeneity in implementing sites is desirable to generate robust clinical and implementation evidence.

\section{Overview of Study Design}

This study will use a Hybrid Type 1 three-arm parallel group randomized controlled design [71] to compare the effectiveness of CETA delivered either in-person or via telephone (T-CETA), compared with a treatment as usual (TAU) control group. CETA trainers (up to $N=6$ ) will be identified from an existing cadre of Zambian counselors and will be trained to be CETA trainers via a technology platform. They will be trained to facilitate technology-delivered CETA trainings to new prospective counselors (up to $N=50$ ) recruited from our partnering organizations, who will be trained to deliver both in-person CETA and TCETA. We will recruit and randomize AYA clients $(\mathrm{N}=400)$ with mental and behavioral health problems from our partnering organizations to one of three conditions: a) CETA, b) T-CETA, or c) TAU. We will evaluate mental and behavioral health outcomes among AYA at baseline, post-treatment (approximately 3-4 months post-baseline), and 6 months post-treatment (approximately 9 months post-baseline) (Fig. 1). Data will also be collected on CETA trainer and counselor competency, fidelity, and knowledge, and implementation constructs (e.g., acceptability, feasibility, cost) associated with training and intervention delivery.

$\ll<$ Insert Fig. 1 here $\gg>$ 


\section{Participants}

AYA participants will be between the ages of 15-29 with mental and behavioral health problems as measured by validated screening tools (see Screening). Trainer and counselor participants will be lay providers interested in mental health training. Full eligibility criteria are summarized in Table 1. 
AYA

\section{15-29 years of age}

2. Attend or be referred to study site

3. Live in the area served by a study site (i.e., not staying temporarily)

4. Ability to speak one of the study languages (English, Bemba, or Nyanja)

5. Screening: Present with one or more common mental/behavioral health problems based on validated screening tools included in the audio computer assisted self-interviewing (ACASI) system. Specifically, the following screening tools and cut-off values:

a. Youth Self Report Internalizing Scale ( $\geq 14)$

b. Youth Self Report Externalizing Scale $(\geq 8)$

c. Child PTSD Symptom Scale $(\geq 12)$

6. Exclusion:

a. Currently on unstable psychiatric drug regimen (e.g., altered in past two months)

b. Suicide attempt or active and severe self-harm in past month

c. Psychotic disorder or severe mental illness

Trainers and Counselors

1. 18 years of age or older

2. Interest in providing CETA

3. Time/availability to participate in the study

4. Minimal education level is comparable to a high school education

5. Ability to speak English fluently and speak at least 1 local language (Nyanja or Bemba)

6. Completion of an in-person interview with study team investigators demonstrating strong communication skills

7. Planning to stay in study area (Lusaka) to provide treatment to clients and/or training to new counselors

Trainers: in addition to all of the above:

8. Interest in teaching CETA

9. Completion of the CETA training

10. Completion of a minimum of 3 CETA cases under supervision 


\section{Recruitment}

For the recruitment of AYA clients, the research team will conduct half-day training sessions explaining CETA, and for whom and what problems it is appropriate, to all staff at collaborating partner organizations. In addition, a separate 2-hour CETA information meeting will be conducted for home-based care workers that help link individuals to services. Recruitment by home-based care workers connected to partner sites is a process used previously by the research group to help identify, connect, and assure that those in need get services, and mirrors how the program could recruit clients in a real-world setting [72, 73]. Initial contact will be made by partner organization staff or home-based care workers who will explain the study with a recruitment script to AYA, and their primary caregiver for those under age 18 , with whom they have previously or currently work. Snowball recruitment methods may also be used to identify additional AYA participants. Recruitment will be conducted in private locations such as a private room in a community location (e.g., church, school) or a private room within the partner organization facilities. AYA that express interest in receiving more information on the study will be connected to the research team.

Trainer and counselor-level participants will be recruited from our collaborating partners and through contacts of local stakeholders like the Ministry of Health to increase the likelihood that provision of CETA continues after the end of the study period. Our collaborating partners and contacts will recommend current staff who they think would be appropriate CETA counselors and have time to provide this service. Potential counselors will also need to submit a resume to the study team, a letter expressing their motivation to be a counselor, and letter of support from their organization. Study staff will interview applicants, and ensure they meet inclusion criteria. Final agreement will be by consensus of study team and collaborating partners.

\section{Informed consent}

Consent activities with all participants involves discussing relevant study details including the purpose, treatment details, privacy, and risks and benefits. Informed consent will be obtained from AYA clients (and caregivers, if applicable) in English, Bemba, or Nyanja by trained research assistants. Consent is obtained for participation in all study activities at the screening phase. AYA will be informed that study participation is voluntary and will not impact any ongoing or future services they may receive. Research assistants will be trained in human subjects and responsible conduct of research and receive appropriate certification. For clients under age 18, research assistants will also obtain informed consent and permission from the AYA's primary caregiver to obtain assent from the AYA participant.

\section{Screening and Baseline Assessment}

The screening portion of the baseline assessment consists of demographic information, internalizing and externalizing symptoms, and posttraumatic stress. Screening instruments will be administered using the audio computer assisted self-interviewing (ACASI) system. Participants can navigate through the questionnaires themselves using a laptop computer while listening to the questions through headphones 
and seeing the question and response options on the screen. A research assistant will be positioned nearby in case the participant has any difficulty. We have used ACASI extensively with AYA populations in Zambia and found it to be an acceptable and feasible approach for measuring mental and behavioral health outcomes. All measures included in the ACASI have previously been used by our team with similar study populations and are available in English, Bemba, and Nyanja [35, 68, 70-72].

Broadband Mental Health Functioning (Youth Self Report (YSR); [77]). The YSR is a 112-item measure of broadband mental health symptoms on a 3-point Likert scale $(0=$ Not True, $1=$ Somewhat True, 2 = Very True or Often True). The measure produces 8 subscales and two broad internalizing and externalizing symptom scores. Eligibility cutoffs of $\geq 14$ and $\geq 8$ will be used for the internalizing and externalizing subscale scores, respectively, based on our previous validity study [75].

Posttraumatic Stress Symptoms (Child PTSD Symptom Scale (CPSS); [78]). The CPSS is a 17-item scale that corresponds to DSM- 5 criteria for PTSD on a 4-point Likert scale $(0=$ Not at all or only one time, $1=$ Once $a$ week or less/once in a while, $2=2$ to 4 times a week/half the time, $3=5$ or more times a week/almost always). Items produce a total Symptom Severity scale. Based on our previous validity study in Zambia, an eligibility cutoff of $\geq 12$ will be used [75].

Participants who do not meet eligibility cutoffs on screening measures will exit the study and be provided resources for mental health and other social services in their area. Eligible AYA participants will continue to complete the second portion of the baseline assessment via the ACASI system, which includes measures of substance use, aggression/violence, functioning, and COVID-19 vaccine hesitancy (see Outcomes).

\section{Randomization and blinding}

Each participant will be assigned a unique ID number by the research assistant during the screening/baseline assessment. Randomization will occur at the individual AYA level and be stratified by site. Following determination of eligibility and completion of the baseline assessment, the research assistant will communicate the ID number to a study staff based at research headquarters who will not interact with study participants. This study staff will maintain a randomization sequence that will be developed a priori by a U.S.-based research staff. This list will not be available or viewable to data collectors in Zambia. The list will have a sequence of treatment assignments (CETA or T-CETA or TAU) in random order, stratified by site, produced via a random number generator in Microsoft Excel. Randomization will be imbalanced (1.5 CETA: 1.5 T-CETA: 1 TAU) due to different sample size requirements in the TAU control arm (see Data Analysis). Eligible AYA participants will be assigned a condition based on the next available slot on the randomization list and inform field staff of the assignment. At this point, field staff will not be blinded to condition assignment for participants they assess. Eligible AYA clients will be informed immediately of the result of the randomization and appropriate next steps: CETA and T-CETA participants will be told that a counselor will be contacting them within 48 hours to setup the first session; TAU participants will be told that the study team will contact them in approximately 3 months to schedule their first post-assessment. The use of ACASI will result in 
the outcomes assessments being blinded; data analysts will also be blind to intervention status when conducting analyses. Due to the nature of the intervention, neither counselors nor AYA clients will be blinded.

\section{Intervention Arms}

CETA

The Common Elements Treatment Approach (CETA) is a transdiagnostic, multi-problem intervention designed to address adult and youth trauma, depression, anxiety, safety, and substance use [79]. It is comprised of a small set of common elements found to be efficacious and prevalent across a range of EBTs to treat common mental health problems. CETA was designed to be flexible in the elements utilized, their order, and their dose (number of sessions) to allow counselors to address heterogeneity, comorbidity, and symptom fluctuations in and across clients. Treatment typically consists of 6 to 12 weekly, approximately 60 -minute sessions delivered by lay workers.

\section{T-CETA}

For adaptation of the CETA manual for telephone delivery, we reviewed evidence-based telehealth strategies and recommendations, telehealth ethical and legal guidelines, and clinical recommendations from telehealth providers. In addition, local trainers-in-training in multiple contexts reviewed telehealth modifications and provided input that was incorporated into the final T-CETA manual used in this study. No changes were made to the structure, duration, and dose of CETA sessions, treatment components, or measurement-based clinical decision-making processes. Telehealth modifications, additions, and strategies were incorporated throughout the manual in delineated "telehealth boxes." This way, the original manual was maintained outside of the telehealth boxes, allowing for clear identification and training of telehealth modifications for both new and existing CETA counselors. A pilot phase was conducted in Zambia to explore the feasibility and acceptability of T-CETA and to obtain feedback for modifying the telehealth additions or implementation strategies. Qualitative results highlighted barriers to T-CETA treatment (i.e., poor connection; client phones off or not having phones; lack of private spaces) and solutions to these barriers (i.e., using community staff's phone for sessions; obtaining multiple contacts for participants; home visits). For those with access to a telephone, T-CETA was deemed by participants as acceptable and effective.

\section{Treatment as usual control}

Participants randomized to the TAU condition will be encouraged to continue engaging with support services normally offered in their community, such as those provided by the partnering organization, and the types and utilization of these services will be tracked. Counselors will check-in with TAU participants monthly to assess safety; any participant with a safety risk will be contacted by a counselor to complete a safety plan and be monitored. All TAU participants will receive a full course of CETA, if desired, after completion of the active study aims. 
CETA training and supervision activities in this study build on the existing apprenticeship model for lay providers to become mental health counselors [80]. In this model, the trainer initially assumes responsibility for teaching core skills and guiding the prospective counselor. After the initial training, supervisors, who may be identified beforehand or from the cohort of new counselors, are responsible for acting as the link between counselors and trainers. Before counselors begin delivering the intervention to clients, supervisors run practice groups with prospective counselors, which are then followed by supervision groups. Practice groups involve role-plays of a particular intervention component or skill, while supervision groups involve each prospective counselor going through one or two pilot cases with a supervisor who has previously piloted the case themselves with a trainer. When supervisors are providing guidance during practice and supervision groups to counselors, supervisors also engage in weekly calls with a trainer to receive consultation and support. At every level, each individual grows in their role over time and develops the necessary skills so that those who deliver coaching and support eventually provide minimal guidance. For this study, trainers-in-training ( $N=$ up to 6$)$ will learn how to train new counselors and supervisors via the technology platform.

The technology-based TTT developed for this study consists of 10 days of trainers-in-training reviewing CETA components on the tech platform, expert CETA trainers modeling training components live via video chat, and live and pre-recorded video observation of trainers-in-training role-plays by expert CETA trainers. Role-plays by trainers-in-training will be rated using a structured rating system, with feedback provided during live video sessions.

Following the TTT, groups of CETA trainers-in-training (pairs, or triplet groups) will each facilitate separate technology-delivered CETA training to the new prospective CETA counselors from our partnering organizations. Trainers-in-training will facilitate technology-delivered trainings to prospective counselors, which include training on standard CETA and T-CETA delivery. Each training will be conducted with approximately 25 counselors ( $N=$ up to 50 ).

\section{Outcomes}

The primary outcomes in the trial will be AYA trauma symptoms (as measured by the CPSS), internalizing symptoms, and externalizing behaviors (both measured by the YSR) administered via ACASI as described in the Screening section. Secondary outcomes among AYA, also administered via ACASI, include substance use, which will be measured with the Alcohol, Smoking, and Substance Involvement Screening Test (ASSIST) $[72,81]$ and aggression/violence as measured by the Youth Victimization Scale [82]. Outcomes are assessed at baseline, post-treatment completion (approximately 3-4 months postbaseline for TAU participants), and 6 months post-treatment completion (approximately 9 months postbaseline for TAU participants). The primary timepoint is post-treatment.

In addition to AYA outcomes, we will collect preliminary data on counselor and trainer competency, fidelity, and knowledge. This will be collected at various points throughout the study through 
standardized role plays and CETA knowledge tests. Mixed methods interviews will be conducted among clients, counselors, and trainers to explore acceptability and feasibility of CETA training and delivery. We will also collect data on health utility among AYA using the EuroQol 5-Dimensions for Youth (EQ-5D-Y) $[83,84]$ to inform the cost-effectiveness analyses. In all, AYA participants will be engaged in the study for approximately 9-12 months.

\section{Data Management}

All data collected on paper forms will include only the participant's ID number. Paper forms will be transferred securely in study vehicles to the storage site where they will be kept in locked filing cabinets within locked offices. Electronic data capture using the ACASI program will also only include participant ID numbers and be stored on encrypted drives.

\section{Data Analysis}

Analysis will be done using an intent-to-treat approach. Mixed effects regression models will be estimated for each mental/behavioral health outcome. Models will include fixed effects of treatment group, time, and a group X time interaction term. Random effects will include client ID and counselor ID. There will be two sets of analyses. First, we will conduct a superiority analysis, in which each of the CETA arms (CETA and T-CETA) is separately compared to TAU. Second, we will conduct a non-inferiority analysis in which we will compare outcomes between the CETA and T-CETA groups.

The total number of AYA participants will be $\mathrm{N}=400$. We conducted power calculations for each of the three primary outcomes for which AYA will be enrolled into the study (trauma symptoms, internalizing symptoms, externalizing behaviors). All three required similar sample sizes; trauma symptoms required the largest sample size and is presented here. Based on a previous study with AYA using the same trauma symptom scale as this study (CPSS) [75], we hypothesize a baseline mean value of approximately 19.0 and standard deviation of 13 . For the superiority analyses, in which we will separately compare each CETA condition (in-person CETA and T-CETA) to the TAU control, we will assume an alpha $=0.025$ to account for two comparisons. Further assuming power $=.80$ and an expected minimum effect size of each CETA condition compared to TAU of 0.5 , we would require 78 persons per arm. For the non-inferiority analysis, in which we will compare the two CETA conditions to each other, we assume an alpha $=0.05$, power $=80 \%$, and a non-inferiority margin of 4.7 , which is equivalent to one-third of the anticipated standard deviation. Previous studies have indicated that differences in outcomes that are less than one third of the standard deviation are considered not practically meaningful. Based on these assumptions we would require 114 in each CETA arm for the non-inferiority comparison. Using the more conservative estimate for the CETA conditions ( $\mathrm{N}=114$ each) and the estimate for the TAU ( $N=78$ ) yields a total sample size of $\mathrm{N}=306$. Assuming $20-25 \%$ attrition based on previous studies, we have inflated the final sample size to $\mathrm{N}=400$ ( $\mathrm{N}=100 \mathrm{TAU}, \mathrm{N}=150 \mathrm{CETA}, \mathrm{N}=150 \mathrm{~T}-\mathrm{CETA})$.

Incremental cost-effectiveness analysis will compare the cost-effectiveness of CETA and T-CETA relative

to TAU. We will first derive quality adjusted life years (QALYs) from health utility reported at each follow 
up time point by AYA, and then estimate differences in mean QALYs gained per treatment condition over study follow up using the mixed effects models detailed above. We will then divide incremental QALYs gained under CETA and T-CETA by the incremental cost of each relative to TAU from the economic perspective of the healthcare provider (i.e., payer's perspective) to estimate the primary cost-effectiveness outcome: incremental cost per QALY gained. Sensitivity analyses will be undertaken to examine uncertainty around the cost-effectiveness estimates, and further interpreted using cost-effectiveness acceptability curves that reveal to decision-makers the probability of the intervention being cost-effective compared to the alternative, given different (implicit monetary) values placed on incremental improvements in the outcome measurement and QALYs. cost-effectiveness acceptability curves will be based on bootstrapped regressions (to account for non-normally distributed data) of study group upon net benefits, controlling for clusters.

\section{Data and safety monitoring}

In addition to ethical review boards, there will be a data and safety monitoring board (DSMB) with researchers from both Zambia and the United States in relevant areas of expertise. Before study initiation, the DSMB will review and approve all study protocols detailing formal procedures for reporting and tracking all adverse reactions, following study progress, and identifying any need for premature termination of the protocol. No interim analyses will be conducted by the DSMB since the intervention is not considered harmful and to avoid erroneous conclusions by running multiple analyses during the study. All major study protocol changes will undergo ethical review and will be updated in the clinical trials registry.

\section{Discussion}

Scalable systems of care, such as those being tested in this trial, are needed to close the mental health treatment gap in LMIC, particularly for populations at-risk for poor outcomes and low treatment adherence, such as adolescents and young adults. The need for telephone-based mental health services in LMIC has become widely discussed during the COVID-19 pandemic. Significant barriers to scale-up of evidence-based treatments in LMIC include a lack of feasible, sustainable training for local lay providers and client access to care. This will be the first CETA trial that is specifically designed to evaluate treatment effectiveness among an adolescent population and the first to feature a technology-based training modality and a telehealth delivery. This study is a first step to build a system of technologydriven training and treatment provision to expand access to evidence based mental healthcare in Zambia and other LMIC settings. The need for effective, remotely delivered interventions is particularly relevant in the COVID-19 era.

There are several notable limitations for this study. First, this trial has a risk of social desirability bias in AYA reporting of symptoms, particularly in the CETA treatment arms given their therapeutic relationship with counselors. To combat this risk, we are using a standardized ACASI assessment system to measure symptoms at all time points, administered by research assistants and not by the counselors themselves. 
Second, this trial is occurring across several urban sites in the capital city of Zambia and our participants will not be representative of AYA in more rural areas of Zambia and across other rural LMIC settings. Additional research will be needed on delivery of these interventions to rural populations where T-CETA may actual be more feasible and acceptable because of access to private space and the high costs of transportation to a clinic.

If the CETA technology training platform is deemed feasible and comparably effective to in-person training models, and T-CETA is found to be as effective as in-person CETA for AYA in Zambia, we aim to expand these technology-driven, cost-effective training and treatment delivery methods across several contexts in sub-Saharan Africa and other LMIC globally.

\section{Abbreviations}

\section{ACASI}

Audio computer assisted self-interviewing system

ASSIST

Alcohol, Smoking and Substance Involvement Screening Test

AYA

Adolescent and young adult

CEACs

Cost-effectiveness acceptability curves

CETA

Common Elements Treatment Approach

CMF

Client Monitoring Form

CPSS

Child PTSD Symptom Scale

DSMB

Data and safety monitoring board

EBT

Evidence-based treatments

EQ-5D-Y

EuroQol 5-Dimensions for Youth

FCV-19S

Fear of COVID-19 Scale

HIC

High-income countries

LMIC

Low- and middle-income countries

NGO

Non-governmental organization 
QALYS

Quality adjusted life years

RCT

Randomized controlled trial

T-CETA

Telehealth-adapted version of CETA

TAU

Treatment as usual

TTT

Train the trainer

YSR

Youth Self Report

\section{Declarations}

\section{Ethical Approval and Consent}

All study procedures were approved by the Institutional Review Board (IRB) of Johns Hopkins University (\#9259/MOD761), and the University of Zambia Biomedical Research Ethics Committee in Zambia (\#011-11-14). All study participants will be provided informed, written consent.

\section{Consent for publication}

Not applicable

\section{Availability of data and materials}

Not applicable

\section{Competing interests}

Not applicable

\section{Funding}

This work is supported by the National Institute of Mental Health (R01MH115495).

\section{Authors' contributions}

CF is co-investigator, primary author and is project director, he helped design the protocol and leads the project. JK is a co-investigator, helped design the protocol, and leads monitoring and evaluation. SS helped design the study protocol and leads project budgeting. EH is co-investigator and helped design the study protocol. MM is lead data specialist, overseeing all M\&E procedures in Zambia. SM is project manager and oversees all project implementation in Zambia. LA helped design the protocol and oversees 
cost effectiveness procedures and analyses. MV is co-investigator and helped design the protocol. AS is co-principal investigator on the trial. SI helped design the protocol. RP helped design the protocol and is a key partner in the Zambia Ministry of Health. FS helped design the protocol and is a key partner in the Zambia Ministry of Health. KM helped design the protocol and led pilot study of this trial. CB is medical director at the partner site and helped design the protocol. CK oversees all cost-effectiveness and implementation measurement and analysis. SB is lead statistician. IS is co-principal investigator and CEO of the partner organization. LM is co-principal investigator, helped design the protocol, and oversees the full project. All authors read and approved the final manuscript.

\section{Acknowledgements}

Authors wish to acknowledge our community partners in Lusaka, Zambia that are critical to the success of this study, including the Centre for Infectious Disease Research in Zambia, YWCA Zambia, Footprint Foundation, NGOCC, Fountain of Hope, Mtendere Health Clinic, and Ngombe Health Clinic.

\section{Trial Status}

This protocol was prospectively registered with www.clinicaltrials.gov (NCT03458039, May 10, 2021). https://clinicaltrials.gov/show/NCT03458039. Protocol Version 6. Recruitment began in October 2021 and is expected to conclude in June 2022.

\section{References}

1. Kieling $\mathrm{C}$, Baker-Henningham $\mathrm{H}$, Belfer $\mathrm{M}$, et al. Child and adolescent mental health worldwide: Evidence for action. The Lancet. Epub ahead of print 2011. DOI: 10.1016/S0140-6736(11)60827-1.

2. Patel V, Kieling C, Maulik PK, et al. Improving access to care for children with mental disorders: A global perspective. Archives of Disease in Childhood. Epub ahead of print 2013. DOI: 10.1136/archdischild-2012-302079.

3. Belfer ML. Child and adolescent mental disorders: the magnitude of the problem across the globe. $J$ Child Psychol Psychiatry. 2008;49:226-36.

4. Kohn R, Saxena S, Levav I, et al. The treatment gap in mental health care. Bull World Health Organ. 2004;82:858-66.

5. Drew N, Funk M, Tang S, et al. Human rights violations of people with mental and psychosocial disabilities: An unresolved global crisis. The Lancet. 2011;378:1664-75.

6. Chisholm D, van Ommeren M, Ayuso-Mateos J-L, et al. Cost-effectiveness of clinical interventions for reducing the global burden of bipolar disorder. Br J Psychiatry. 2005;187:559-67.

7. Thornicroft G. Physical health disparities and mental illness: The scandal of premature mortality. $\mathrm{Br}$ J Psychiatry. 2011;199:441-2.

8. McDaid D, Knapp M, Raja S. Barriers in the mind: promoting an economic case for mental health in low- and middle-income countries. World Psychiatry. 2008;7:79-86. 
9. Kalichman SC, Simbayi LC, Vermaak R, et al. Randomized trial of a community-based alcohol-related HIV risk-reduction intervention for men and women in Cape Town South Africa. Ann Behav Med. 2008;36:270-9.

10. Murray CJL, Vos T, Lozano R, et al. Disability-adjusted life years (DALYs) for 291 diseases and injuries in 21 regions, 1990-2010: A systematic analysis for the Global Burden of Disease Study 2010. Lancet. 2012;380:2197-223.

11. Joint United Nations Programme on HIV/AIDS (UNAIDS). Young People and HIV. 2021.

12. Central Statistical Office. Zambia: 2010 Census of Population and Housing. 2011.

13. Central Statistical Office Zambia. Zambia Demographic and Health Survey 2013-3014. 2014.

14. Zambia, Demographic and Health Survey 2013-2014. 2014.

15. Benjet C. Childhood adversities of populations living in low-income countries: prevalence, characteristics, and mental health consequences. Curr Opin Psychiatry. 2010;23:356-62.

16. Monasch R, Boerma JT. Orphanhood and childcare patterns in sub-Saharan Africa. AIDS. 2004;18:55-65.

17. Andrews G, Skinner D, Zuma K. Epidemiology of health and vulnerability among children orphaned and made vulnerable by HIV/AIDS in sub-Saharan Africa. AIDS Care. 2006;18:269-76.

18. Cluver L, Gardner F. Risk and protective factors for psychological well-being of children orphaned by AIDS in Cape Town: a qualitative study of children and caregivers' perspectives. AIDS Care; 19. Epub ahead of print March 2007. DOI: 10.1080/09540120600986578.

19. Cluver LD, Orkin M, Gardner F, et al. Persisting mental health problems among AIDS-orphaned children in South Africa. J Child Psychol Psychiatry Allied Discip. 2012;53:363-70.

20. Cluver L, Gardner F. The mental health of children orphaned by AIDS: a review of international and southern African research. J Child Adolesc Ment Heal. 2007;19:1-17.

21. Kaplow JB, Saunders J, Angold A, et al. Psychiatric symptoms in bereaved versus nonbereaved youth and young adults: A longitudinal epidemiological study. J Am Acad Child Adolesc Psychiatry. 2010;49:1145-54.

22. Gregson S, Nyamukapa C, Garnett GP, et al. HIV infection and reproductive health in teenage women orphaned and made vulnerable by AIDS in Zimbabwe. AIDS Care. 2005;17:785-94.

23. Reza A, Breiding MJ, Gulaid J, et al. Sexual violence and its health consequences for female children in Swaziland: a cluster survey study. Lancet. 2009;373:1966-72.

24. Stöckl H, Kalra N, Jacobi J, et al. Is early sexual debut a risk factor for HIV infection among women in sub-Saharan Africa? A systematic review. Am J Reprod Immunol. 2013;69(Suppl 1):27-40.

25. Bass JK, Annan J, Murray SMI, et al. Controlled trial of psychotherapy for congolese survivors of sexual violence. N Engl J Med. Epub ahead of print 2013. DOI: 10.1056/NEJMoa1211853.

26. Bolton P, Bass J, Neugebauer R, et al. Group interpersonal psychotherapy for depression in rural Uganda: a randomized controlled trial. JAMA. 2003;289:3117-24. 
27. Rahman A, Malik A, Sikander S, et al. Cognitive behaviour therapy-based intervention by community health workers for mothers with depression and their infants in rural Pakistan: a cluster-randomised controlled trial. Lancet. 2008;372:902-9.

28. Patel V, Weiss HA, Chowdhary N, et al. Effectiveness of an intervention led by lay health counsellors for depressive and anxiety disorders in primary care in Goa, India (MANAS): A cluster randomised controlled trial. Lancet. 2010;376:2086-95.

29. Murray LK, Skavenski S, Kane JC, et al. Effectiveness of Trauma-Focused Cognitive Behavioral Therapy Among Trauma-Affected Children in Lusaka, Zambia. JAMA Pediatr. 2015;169:761.

30. Bolton P, Bass J, Betancourt T, et al. Interventions for depression symptoms among adolescent survivors of war and displacement in northern Uganda: a randomized controlled trial. JAMA J Am Med Assoc. 2007;298:519-27.

31. Murray LK, Skavenski S, Michalopoulos LM, et al. Counselor and Client Perspectives of TraumaFocused Cognitive Behavioral Therapy for Children in Zambia: A Qualitative Study. J Clin Child Adolesc Psychol. Epub ahead of print January 2014. DOI: 10.1080/15374416.2013.859079.

32. Patel V, Chowdhary N, Rahman A, et al. Improving access to psychological treatments: lessons from developing countries. Behav Res Ther. 2011;49:523-8.

33. Chorpita BF, Daleiden EL, Weisz JR. Identifying and selecting the common elements of evidence based interventions: A distillation and matching model. Ment Health Serv Res. 2005;7:5-20.

34. Weiss WM, Murray LK, Zangana GAS, et al. Community-based mental health treatments for survivors of torture and militant attacks in Southern Iraq: A randomized control trial. BMC Psychiatry. Epub ahead of print 2015. DOI: 10.1186/s12888-015-0622-7.

35. Bolton P, Lee C, Haroz EE, et al. A Transdiagnostic Community-Based Mental Health Treatment for Comorbid Disorders: Development and Outcomes of a Randomized Controlled Trial among Burmese Refugees in Thailand. PLoS Med; 11. Epub ahead of print 2014. DOI: 10.1371/journal.pmed.1001757.

36. Murray LK, Kane JC, Glass N, et al. Effectiveness of the Common Elements Treatment Approach (CETA) in reducing intimate partner violence and hazardous alcohol use in Zambia (VATU): A randomized controlled trial. PLoS Med. 2020;17:1-23.

37. Bonilla-Escobar FJ, Fandiño-Losada A, Martínez-Buitrago DM, et al. A randomized controlled trial of a transdiagnostic cognitive-behavioral intervention for Afro-descendants' survivors of systemic violence in Colombia. PLoS ONE. 2018;13:e0208483.

38. Bogdanov S, Augustinavicius J, Bass JK, et al. A randomized controlled trial of community-based transdiagnostic psychotherapy for veterans and internally displaced persons in Ukraine.

39. Kane JC, Sharma A, Murray LK, et al. Effectiveness of the Common Elements Treatment Approach (CETA) for unhealthy alcohol use among adults with HIV in Zambia: Results from a pilot randomized controlled trial.

40. Murray LK, Hall BJ, Dorsey S, et al. An evaluation of a common elements treatment approach for youth in Somali refugee camps. Glob Ment Heal (Cambridge, England) 2018; 5: e16. 
41. Murray LK, Dorsey S, Bolton P, et al. Building capacity in mental health interventions in low resource countries: An apprenticeship model for training local providers. Int J Ment Health Syst. 2011;5:30.

42. Assemi M, Mutha S, Hudmon KS. Evaluation of a train-the-trainer program for cultural competence. Am J Pharm Educ; 71. Epub ahead of print 2007. DOI: 10.5688/aj7106110.

43. Corelli RL, Fenlon CM, Kroon LA, et al. Evaluation of a train-the-trainer program for tobacco cessation. Am J Pharm Educ; 71. Epub ahead of print 2007. DOI: 10.5688/aj7106109.

44. Nyamathi A, Vatsa M, Khakha DC, et al. HIV knowledge improvement among nurses in India: using a train-the-trainer program. J Assoc Nurses AIDS Care. 2008;19:443-9.

45. Tanabe P, Stevenson A, DeCastro L, et al. Evaluation of a train-the-trainer workshop on sickle cell disease for ED providers. J Emerg Nurs. 2013;39:539-46.

46. Martino S, Ball SA, Nich $\mathrm{C}$, et al. Teaching community program clinicians motivational interviewing using expert and train-the-trainer strategies. Addiction. 2011;106:428-41.

47. Chamberlain P, Price J, Reid J, et al. Cascading implementation of a foster and kinship parent intervention. Child Welfare. 2008;87:27-48.

48. Carruth AK, Pryor S, Cormier C, et al. Evaluation of a School-Based Train-the-Trainer Intervention Program to Teach First Aid and Risk Reduction Among High School Students. J Sch Health. 2010;80:453-60.

49. Pearce J, Mann MK, Jones C, et al. The most effective way of delivering a Train-the-Trainers program: A systematic review. J Continuing Educ Health Professions. 2012;32:215-26.

50. Chandar U, Sharma R. Bridges to effective learning through radio. Int Rev Res Open Distance Learn. 2003;4:np.

51. Mishra S. Audio, radio and interactive radio. Educ Media Asia; 71.

52. Tripp SD, Roby WB. Auditory presentations and language laboratories. In: Jonassen DH, editor. Handbook ofResearchfor Educational Communications and Technology. New York: Macmillan; 1996. pp. $821-50$.

53. Lappia A, Kirkland J. Audio-cassette Tapes in Distance Teaching: Student Evaluation. Distance Educ. 1989;10:277-84.

54. Kakuma R, Minas H, Van Ginneken N, et al. Human resources for mental health care: Current situation and strategies for action. The Lancet. 2011;378:1654-63.

55. Ray ML, Wilson MM, Wandersman A, et al. Using a training-of-trainers approach and proactive technical assistance to bring evidence based programs to scale: an operationalization of the interactive systems framework's support system. Am J Community Psychol. 2012;50:415-27.

56. Acharibasam JW, Wynn R. Telemental Health in Low-and Middle-Income Countries: A Systematic Review. Epub ahead of print 2018. DOI: 10.1155/2018/9602821.

57. Huang K-Y, Lee D, Nakigudde J, et al. Use of Technology to Promote Child Behavioral Health in the Context of Pediatric Care: A Scoping Review and Applications to Low- and Middle-Income Countries. Front Psychiatry. 2019;10:806. 
58. Malhotra S, Shah R. Telepsychiatry and Digital Mental Health Care in Child and Adolescent Psychiatry Implications for Service Delivery In Low- and Middle-Income Countries. In: Understanding Uniqueness and Diversity in Child and Adolescent Mental Health. Elsevier; 2018. pp. 263-87.

59. Myers K, Valentine J, Morganthaler R, et al. Telepsychiatry with incarcerated youth. J Adolesc Heal. 2006;38:643-8.

60. Myers K, Valentine JM, Melzer SM. Feasibility, acceptability, and sustainability of telepsychiatry for children and adolescents. Psychiatr Serv. 2007;58:1493-6.

61. Kopel H, Nunn K, Dossetor D. Evaluating satisfaction with a child and adolescent psychological telemedicine outreach service. J Telemed Telecare. 2001;7:35-40.

62. Elford DR, White $\mathrm{H}$, St John $\mathrm{K}$, et al. A prospective satisfaction study and cost analysis of a pilot child telepsychiatry service in Newfoundland. J Telemed Telecare. 2001;7:73-81.

63. Salum GA, Rehmenklau JF, Csordas MC, et al. Supporting people with severe mental health conditions during the COVID-19 pandemic: considerations for low- and middle-income countries using telehealth case management. Revista brasileira de psiquiatria (Sao Paulo, Brazil: 1999) 2020; 42: 451-452.

64. Hungerbuehler I, Valiengo L, Loch AA, et al. Home-Based Psychiatric Outpatient Care Through Videoconferencing for Depression: A Randomized Controlled Follow-Up Trial. JMIR Ment Heal. 2016;3:e36.

65. Naal H, Whaibeh E, Mahmoud H. International Review of Psychiatry Guidelines for primary health care-based telemental health in a low-to middle-income country: the case of Lebanon. Int Rev Psychiatry. Epub ahead of print 2020. DOI: 10.1080/09540261.2020.1766867.

66. Thara R, John S, Rao K. Telepsychiatry in Chennai, India: The SCARF experience. Behav Sci Law. 2008;26:315-22.

67. Tharoor H, Thara R. Evolution of Community Telepsychiatry in India Showcasing the SCARF Model. Indian J Psychol Med. 2020;42:69S-74S.

68. Sanchez T, Peña E, Ng B. Mental health in the age of COVID-19, a Mexican experience. Indian J Psychiatry. 2020;62:377-9.

69. World Bank Group. Mobile cellular subscriptions (per 100 people) - Zambia.

70. Naskar S, Victor R, Das H, et al. Telepsychiatry in India-Where do we stand? A comparative review between global and Indian telepsychiatry programs. Indian J Psychol Med. 2017;39:223-42.

71. Curran GM, Bauer M, Mittman B, et al. Effectiveness-implementation Hybrid Designs. Med Care. 2012;50:217-26.

72. Kane JC, Murray LK, Bass JK, et al. Validation of a substance and alcohol use assessment instrument among orphans and vulnerable children in Zambia using Audio Computer Assisted SelfInterviewing (ACASI). Drug Alcohol Depend. 2016;166:85-92.

73. Murray LK, Kane JC, Glass N, et al. Effectiveness of the Common Elements Treatment Approach (CETA) in reducing intimate partner violence and hazardous alcohol use in Zambia (VATU): A 
randomized controlled trial. PLOS Med. 2020;17:e1003056.

74. Kane JC, Bolton PA, Murray SM, et al. Psychometric evaluation of HIV risk behavior assessments using Audio Computer Assisted Self-Interviewing (ACASI) among orphans and vulnerable children in Zambia. AIDS Care. 2018;30:160-7.

75. Murray SM, Bolton P, Kane JC, et al. Measuring Symptoms of Psychopathology in Zambian Orphans and Vulnerable Children: Scale Validation and Psychometric Evaluation. Assessment 2018; 107319111878045.

76. Kane JC, Murray LK, Sughrue S, et al. Process and implementation of Audio Computer Assisted SelfInterviewing (ACASI) assessments in low resource settings: a case example from Zambia. Glob Ment Heal. 2016;3:e24.

77. Achenbach TM. The Achenbach System of Empirically Based Assessemnt (ASEBA): Development, Findings, Theory, and Applications. Burlington: University of Vermont Research Center for Children, Youth, \& Families; 2009.

78. Foa EB, Johnson KM, Feeny NC, et al. The Child PTSD Symptom Scale: A Preliminary Examination of its Psychometric Properties. J Clin Child Adolesc Psychol. 2001;30:376-84.

79. Murray LK, Dorsey S, Haroz E, et al. A common elements treatment approach for adult mental health problems in low- and middle-income countries. Cogn Behav Pract. 2014;21:111-23.

80. Murray LK, Dorsey S, Bolton P, et al. Building capacity in mental health interventions in low resource countries: an apprenticeship model for training local providers. Int J Ment Health Syst; 5 . Epub ahead of print September 2011. DOI: 10.1186/1752-4458-5-30.

81. WHO ASSIST Working Group. The Alcohol, Smoking and Substance Involvement Screening Test (ASSIST): development, reliability and feasibility. Addiction. 2002;97:1183-94.

82. Nadel H, Spellmann M, Alvarez-Canino T, et al. The Cycle of Violence and Victimization: A Study of the School-based Intervention of a Multidisciplinary Youth Violence-Prevention Program. Am J Prev Med. 1996;12:109-19.

83. Wille N, Badia X, Bonsel G, et al. Development of the EQ-5D-Y: a child-friendly version of the EQ-5D. Qual Life Res; 19. Epub ahead of print August 2010. DOI: 10.1007/s11136-010-9648-y.

84. Ravens-Sieberer U, Wille N, Badia X, et al. Feasibility, reliability, and validity of the EQ-5D-Y: results from a multinational study. Qual Life Res. 2010;19:887-97.

\section{Figures}


Figure 1. Spirit flow diagram of trial schedule

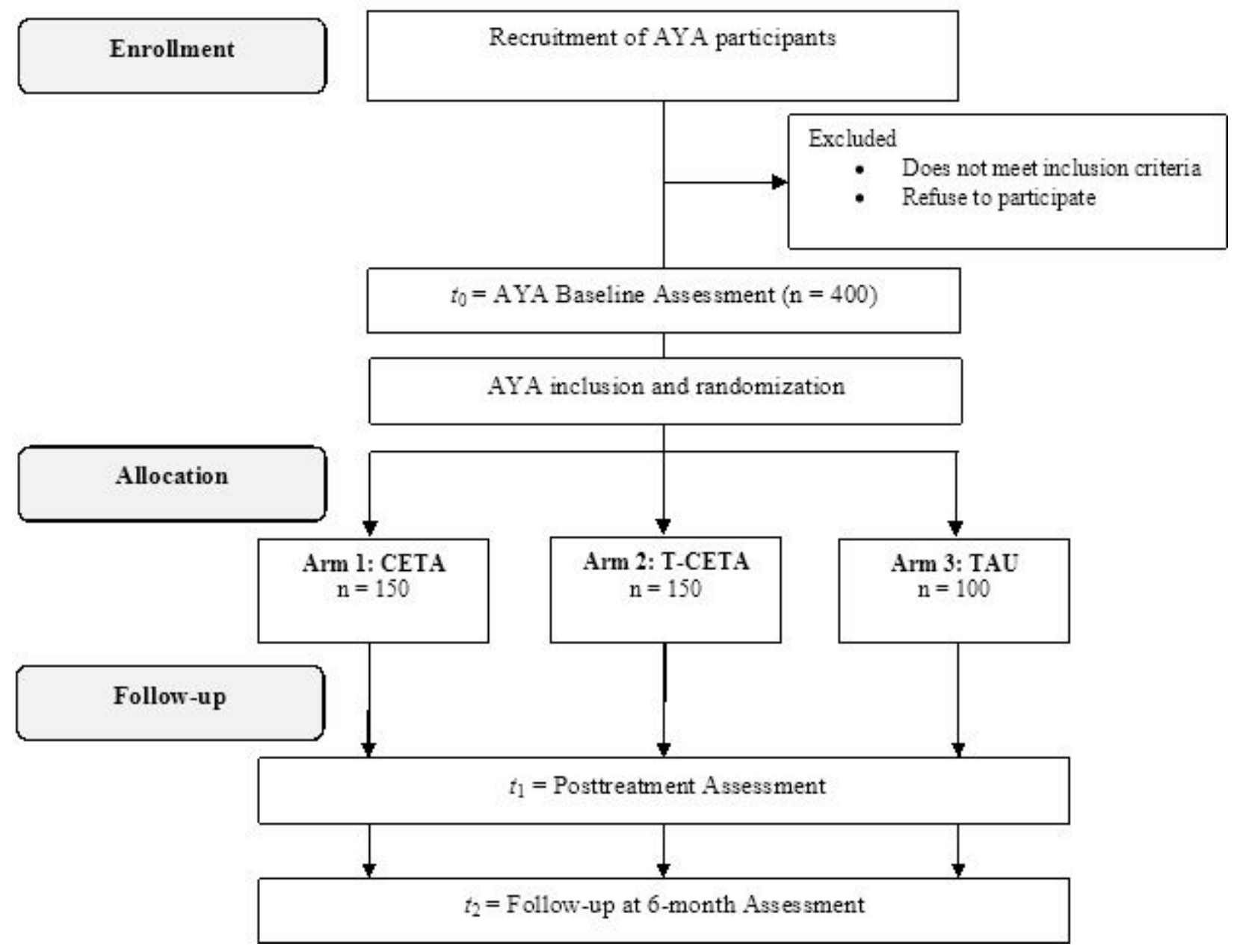

Figure 1

Spirit flow diagram of trial schedule

\section{Supplementary Files}

This is a list of supplementary files associated with this preprint. Click to download.

- SPIRITChecklist.doc 